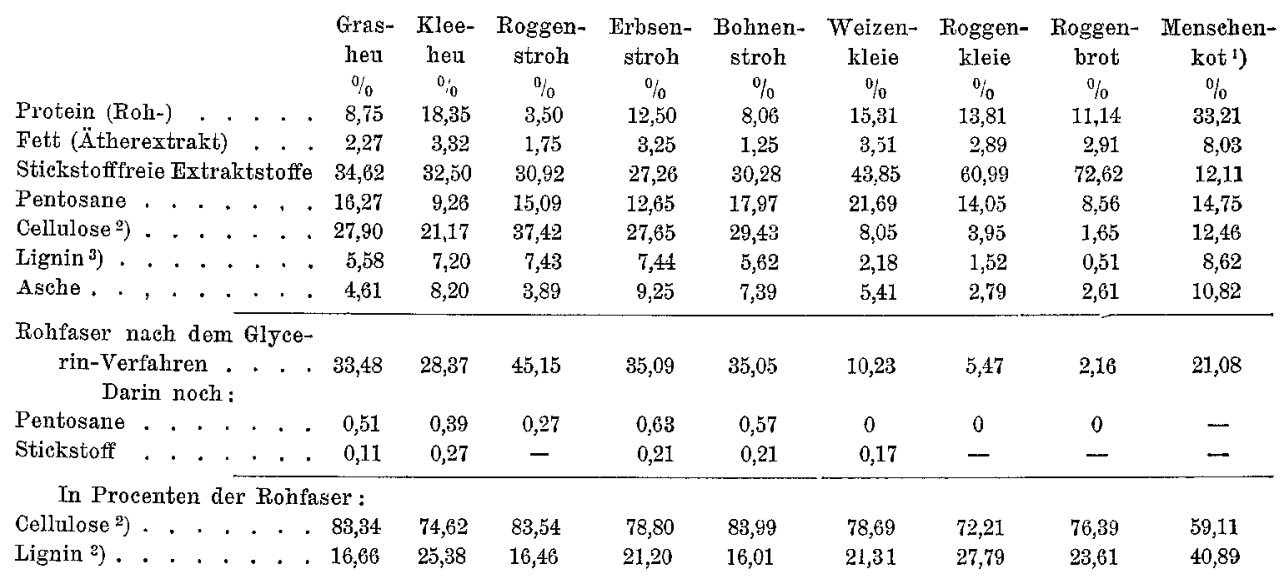

1) Nach einer Kost von vorwiegend Roggenbrot, Erbsen, Bohnen und Speck.

$\left.{ }^{2}\right)$ d. h. Rückstand von der Oxydation mit Wasserstoffsuperoxyd und Ammoniak.

$\left.{ }^{3}\right)$ d. h. oxydierter Teil von der Oxydation mit Wasserstoffsuperoxyd und Ammoniak.

Mïgen diese Zahlen wegen des noch nicht genügend ausgebildeten Verfahrens auch verbesserungsbedürftig sein, so sehen wir doch daraus, daß die Futter- und Nahrungsmittel sich im Gehalt an Cellulose (schwer oxydierbarem Rückstand) im Verhältnis zur Rohfaser nicht unwesentlich verschieden verhalten, und daß von der Rohfaser sich vorwiegend das Lignin im Kot ansammelt.

Jedenfalls wird ein Verfahren zur quantitativen Bestimmung der Cellulose und des Lignins in den Futter- und Nahrungsmitteln von nicht geringem Wert für die Beurteilung derselben sein, da die bisherigen, am Tiere wie Menschen angestellten Versuche schon im allgemeinen erkennen lassen, daß die Ausnutzungsfähigkeit der Cellulose derselben mit dem Lignin-Gehalt steigt und fällt, d. h. um so höher sich stellt, je weniger Lignin vorhanden ist und umgekehrt.

Auch hierüber sollen weitere Untersuchungen noch sicherere Aufschlüsse bringen.

\title{
Untersuchung von Fleisch-, Hefen- und anderen Extrakten auf Xanthinkörper.
}

\section{Die Xanthinkörper des Fleischextraktes. Von}

\section{Dr. Karl Micko.}

Mitteilungen aus der staatlichen Untersuchungsanstalt für Lebensmittel in Graz.

Im vorigen Jahrgange dieser Zeitschrift ${ }^{1}$ ) veröffentlichte ich eine Abhandlung über eine vergleichende Untersuchung von Fleischextrakten und deren Ersatzmitteln.

1) Diese Zeitschrift 1902, 5, 193. 
Dieselbe befaßte sich zum Teil mit der Bestimmung des Gehaltes mehrerer Extrakte an Xanthinkörper-Stickstoff und es konnte auf Grund der gewonnenen Ergebnisse deutlich die abweichende Zusammensetzung der organischen Substanz mancher der untersuchten Präparate im Vergleich zu der des Fleischextraktes gezeigt werden. In einem der untersuchten Präparate wurden nämlich nur geringe Mengen Xanthinstoffe vorgefunden. In anderen Präparaten, wie in den Hefenextrakten, war der Gehalt an Xanthinkörper-Stickstoff auffällig hoch. Ich habe nun die Untersuchungen über das Vorkommen der Xanthinstoffe in Extrakten weiter ausgedehnt und suchte die einzelnen Xanthinkörper zu isolieren und zu kennzeichnen. Es war nämlich zu erwägen, ob sich in den verschiedenen Präparaten stets dieselben Xanthinstoffe finden, ob ihr gegenseitiges Mengenverhältnis dasselbe ist, oder ob bestimmte Xanthinstoffe das eine oder das andere Präparat charakterisieren, indem dieselben in den übrigen Präparaten nicht oder nur in geringer Menge vorkommen. Denn es war nicht unwahrscheinlich, daß die Erkennung der einzelnen Xanthinstoffe verwendbare Anhaltspunkte über die Herkunft eines fraglichen Präparates ergeben kann. Wie sehr dies zutrifft, wird in der nächsten Mitteilung „Die Xanthinkörper der Hefenextrakte" näher bewiesen und des weiteren gezeigt werden, daß ein gewaltiger Unterschied in der Zusammensetzung der organischen Substanz der Hefenextrakte im Vergleich zu der des Fleischextraktes besteht.

$\mathrm{Zu}$ den bekannten im Fleischextrakt vorkommenden Xanthinbasen gehören das Hypoxanthin, das Xanthin und das von Weidel entdeckte Karuin. Mitteilungen über eine systematische Untersuchung des Fleischextraktes auf Xanthinstoffe fand ich in der Literatur nicht vor. Eine derartige Untersuchung ersehien umso wünschenswerter, als in der bereits erwäbnten Abhandlung nachgewiesen wurde, daß behufs vollständiger Abscheidung der Xanthinstoffe das vorherige Kochen des Extraktes mit verdünnter Säure notwendig ist. Denn es könnte vorkommen, daß ohne diese Behandlung Xanthinstoffe, die erst aus ibren Verbindungen abgespalten werden müssen, sich dem Nachweise entziehen. Zur Abscheidung der Xanthinbasen bediente ich mich desselben Verfahrens, wie bei der Bestimmung des Gesamt-Xanthinkörper-Stickstoffes.

Der Extrakt wurde zunächst nit verdünnter Säure gekocht und aus der erhaltenen Lösung wurden die Xanthiukörper nach dem Kr üger'schen Verfahren mit Kupferbisulfit gefällt und schließlich aus ihren unlöslichen Silberverbindungen als salzsaure Salze gewonnen. Zur weiteren Verarbeitung der erhaltenen Präparate benutzte ich im Principe das Verfahren, dessen sich Krüger und Salom on ${ }^{1}$ ) zur Identifizierung der Alloxurbasen im Harn bedient haben. Die beiden Autoren vermieden die Anwendung stärkerer Salpetersäure zur Isolierung fer einzelnen Xanthinbasen. Ich ging der Anwendung der Salpetersäure noch mehr aus dem Wege und beschränkte dieselbe nur auf die Untersuchung von kleineren Fraktionen. Es war nämlich Rücksicht zu nehmen auf die wenig bekannte Base, - das Karnin - und es wäre nicht ausgeschlossen, dab die Salpetersäure wenigetens teilweise zersetzend auf das Karnin wirken könnte. Für die Ausführung der nachstehenden Untersuchungen verwendete ich eine größere Menge Extrakt, welche mir von der Liebig's Fleischextrakt-Kompagnie freundlichst zur Verfügung gestellt wurde.

Der Gang der Untersuchung gestaltete sich, wie folgt:

Je $300 \mathrm{~g}$ Extrakt (jm ganzen $3000 \mathrm{~g}$ Extrakt) wurden in $2250 \mathrm{~cm}$ Wasser

1) Zeitschr. physiol. Chem. 1898, 26, 350. 
und $250 \mathrm{ccm}$ verdünnter Schwefelsäure $(1: 3)$ gelöst. Nach dreistündigem Kochen neutralisierte ich die saure Lösung mit Natronlauge und fällte die Xanthinstoffe in der Siedehitze mit je $900 \mathrm{ccm}$ Natriumbisulfitlösung (200 $\mathrm{g}$ Natriumbisulfit in Pulver auf 1 I Wasser) und Kupfersulfatlösung (130 g auf 1 l Wasser). Der am nächsten Tage abfiltrierte und mit Wasser gewaschene Niederschlag wurde in einen Kolben eingetragen, mit Wasser und verdünnter Salzsäure, letztere in entsprechendem Überschuß, vermischt. Das Ausfällen von Kupfer aus dieser Flüssigkeit mit Schwefelwasserstoff geschah im heißen Wasserbade, um das Ubergehen der etwa sehr schwer löshichen Xanthinhasen in das niederfallende Schwefelkupfer zu verhüten. In dem eingedampften Filtrate vom Schwefelkupfer entatand auf Zusatz von Ammoniak in mäßigem Überschuß nach mehrstündigem Stehen ein unbedentender dunkel gefärbter Niederschlag, der hauptsächlich aus Calciumphosphat bestand und auBerdem aber anch Eisen enthielt. Das Filtrat von diesem Niederschlage wurde in eine ammoniakalische Silberlösung in dünnem Strahle und unter beständigem Rübren eirgegossen. Den entstandenen, nach mehreren Stunden abfiltrierten Niederschlag wusch ich zunächst mit stark verdünntem Ammoniak, dann mit Wasser so lange, bis das Filtrat keine Salpetersäure-Reaktion mit Diphenylamin gab, schwemmte denselben sodann in heißem Wasser unter Zusatz von verdünnter Salzsäure auf und leitete in die Flüssigkeit Schwefelwasserstoff ein. Dem abfiltrierten Schwefelsilber konnten durch Erhitzen mit verdünnter Salzsäure keine Xanthinkörper mehr entzogen werden. Das Filtrat vom Schwefelsilber wurde zunächst in einer Porzellanschale über freiem Feuer unter Benutzung eines Drahtnetzes, dann aber im Vakuum zur Vertreibung der überschüssigen Salzsäure eingedampft. Der Vakuum-Rückstand löste sich leicht in Wasser. Da die Lösung braun gefärbt war, entfürbte ich dieselbe mit möglichst wenig Tierkohle und zwar in der Hitze. Während dieser Behandlung schien die Tierkohle merklicho Mengen der Xanthinkörper zurückzuhalten. Ich habe deshalb ungefähr die Hälfte der gebrauchten Tierkohle mit verdünnter Salzsäure mehrere Stunden lang auf dem Wasserbade erwärmt. Aus dem Filtrate konnte ich noch $1 \mathrm{~g}$ auf dem Wasserbade getrocknete Xanthinstoffe, bezw. deren salzsaure Salze gewinnen. Die Entfärbung der Xanthinkörperlösungen mit Tierkohle, selbst in der Wärme, ist demnach zu verwerfen. Es empfiehlt sich vielmehr zu diesem $Z$ wecke ein weiter unten beschriebenes Entfärbungsverfahren der siedend heißen Lösungen mit Bleiessig unter Zusatz von Ammoniak. Die mit Tierkohle entfärbten Lösungen wurden auf dem Wasserbade bis zur Trockne eingedamptt, der Trockenrückstand in Wasser aufgenommen und die Lösung abermals his zur Trockne eingedampft. Der nun erhaltene Rückstand löste sich in einer entsprechenden Menge Wasser bei 40 bis $50^{\circ} \mathrm{C}$. fast vollständig auf und konnte demnach keine größeren Mengen Xanthin enthalten. Nach dem Erkalten und mehrstündigem Stehen schied sich aus der Flüssigkeit ein pulveriger Bodensatz ab. Es wurde bei diesem Lösungsverfahren soviel Wasser verwendet, daß sich nach dem Abkühlen der Lösung die Xanthinstoffe nicht als salzsaure Salze in Krystallform abgeschieden haben. Die abfiltrierte, mit kaltem Wasser und Alkohol gewaschene und bei $100^{\circ} \mathrm{C}$. getrocknete unlösliche Substanz wog $17 \mathrm{~g}$ (Fraktion A). Der aus den eingedampften Filtraten erhaltene Rückstand lieferte, der eben beschriebenen Behandlung unterworfen, noch 7,5 g unlöslicher Substanz (Fraktion B). Die beiden Fraktionen schieden sich offenbar durch die allmähliche Dissociation ihrer salzsauren Salze ab, denn die Filtrate von denselben enthielten stets überschüssige Salzsäure. Da ich befürchtete, daß durch wiederholtes Eindampfen der Lösungen zur Trockne 
sich schließlich auch die beständigeren Salze der Xanthinbasen teilweise dissoziierten und in das Unlösliche übergehen würden, welche Befürchtung sich übrigens im Laufe der Untersuchung als unbegründet erwies, unterlieb ich es, dieses Verfahren zum dritten Male zu wiederholen. Der zur Trockne durch Eindampfen des Filtrates von der Fraktion B erhaltene Rückstand ist als Fraktion $\mathrm{O}$ bezeichnet.

\section{Untersuchung der Fraktion A.}

Das Präparat löste sich nicht sehwer in heißem Wasser auf. Mit konz. Salpetersäure eingedampft und mit Natronlauge befeuchtet gab der Rückstand zwar eine merkliche, aber keineswegs stärkere Xanthinreaktion. Die Substanz löste sich in verdünntem Ammoniak beim Erwärmen und ebenso in der 15-fachen Menge 3,3\%-iger Natronlauge leicht auf. Die Lösungen schieden selbst nach 24-stündigem Stehen keine unlöslichen Substanzen ab. Die wässerige Lösung der Substanz gab mit Pikrinsäure keinen Niederschlag. Erst bei langsamer Verdunstung schieden sich aus dieser Lösung derbe, in warmem Wasser leicht lösliche Krystalle ab. Ich suchte nun das Präparat durch fraktionierte Krystallisation zu reinigen, kam damit jedoch nicht zu dem erwünschten Ziele, da das Präparat hartnäckig andere Substanzen zurïckhielt, welche dasselbe gelblich färbten. Zur Reinigung dieser Fraktion benutzte ich folgendes Verfahren, welches sich auch bewährte: Eine siedend heiße Lösung von je $3 \mathrm{~g}$ in etwa $1200 \mathrm{ccm}$ Wasser wurde mit $40 \mathrm{cem}$ Bleiessig versetzt. Die Lösung blieb unverändert. Es wurde nun so viel Ammoniak tropfenweise zugesetzt, bis die Flüssigkeit nach dem Absetzen des schmutzig gefärbten Bleiniederschlages entfärbt erschien. Nach dem Entbleien des Filtrates von dem mit heifem Wasser gewaschenen Bleiniederschlage $\left(\mathrm{Pb}_{1}\right)$ mit Schwefelwasserstoff, - wobei der Kolben in ein heißes Wasserbad gesetzt wurde, - war die vom Schwefelblei abfiltrierte Flüssigkeit vollständig entfärbt. Aus derselben schied sich beim Eindampfen bis auf ein kleines Volumen eine fast rein weibe krystallinische Substanz ab, welche nach dem Abfiltrieren und Waschen mit kaltem Wasser nochmals in siedendem Wasser gelöst wurde. Während des Eindampfens dieser Lösung entnahm ich zeitweise Proben von der sich ausscheidenden weißen Substanz und untersuchte dieselbe mikroskopisch. Sie bestand aus zumeist zu Krusten vereinigten, sparrig angeordneten, zugespitzten Krystallen, augenscheinlich quadratischen Oktaedern mit oft krummen Flächen. Die Analyse des mit Wasser und Alkohol gewaschenen und bei $130^{\circ} \mathrm{C}$. getrockneten Präparates lieferte folgendes Ergebnis:

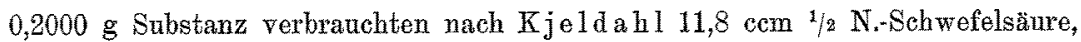
$0,2755 \mathrm{~g}$. gaben $0,07 \mathrm{~g}$ Wasser und $0,448 \mathrm{~g}$ Kohlensäure; demnach:

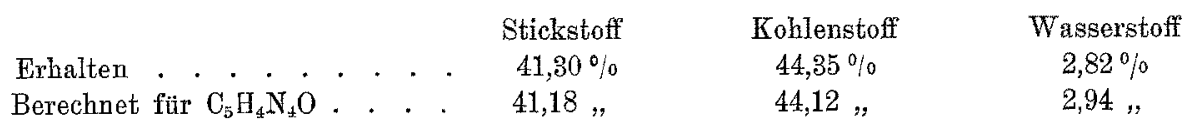

$1 \mathrm{~g}$ des Präparates führte ich in das salpetersaure Salz über, welches bei der mikroskopischen Untersuchung eine Einheitlichkeit der Krystallformen ergab; somit waren dem Salze keine fremden krystallinischen Substanzen beigemischt. Das salpetersaure Salz wurde in Wasser gelöst und die Lösung mit überschüssigem Ammoniak versetzt. Beim Eindampfen der Flüssigkeit und in dem Maße als das überschüssige Ammoniak entwich, schied sich die ursprüngliche Substanz wieder aus, welche auf einem Filter gesammelt, mit Wasser und Alkohol gewaschen und schließlich bei $130^{\circ} \mathrm{C}$. getrocknet, folgenden Stickstoffgehalt aufwies: 
$0,2 \mathrm{~g}$ Substanz verbrauchten nach $\mathrm{Kjel} \mathrm{dah} 111,8 \mathrm{ccm}{ }^{1 / 2} \mathrm{~N}$-Schwefelsäure entsprechend einem Stickstoff-Gehalt von $41,8 \%$.

In einem aus einem anderen Teile der Fraktion A gewonnenen Präparate bestimmte ich gleichfalls den Gehalt an Stickstoff.

0,2 g Substanz verbrauchten nach Kjeldahl $11,8 \mathrm{ccm}{ }^{1} / 2$ N.-Schwefelsäure entsprechend einem Stickstoff-Gehalt ron $41,3 \%$;

Nicht nur das analytische Ergebnis, sondern auch das sonstige Verhalten der untersuchten Substanzen beweist, daß die Substanzen mit Hypoxanthin identisch sind.

Der bleihaltige Niederschlag $\mathrm{Pb}_{1}$ wurde in siedend heiBem Wasser aufgeschwemmt und mit Essigsäure in Lösung gebracht. Zu dieser Lösung setzte ich noch Bleiessig zu, hierauf Ammoniak bis zur Entfärbung der Flüssigkeit. Der entstandene Niederschlag ist mit $\mathrm{Pb}_{2}$ bezeichnet. Die aus dem mit Schwefelwasserstoff entbleiten und eingeengten Filtrate gewonnene Substanz betrug $0,86 \mathrm{~g}$.

$0,2 \mathrm{~g}$ dieser bei $130^{\circ} \mathrm{C}$. getrockneten Substanz verbrauchten nach KjeIdah $111,15 \mathrm{ccm}$ $1 / 2$ N.-Schwefelsätre. Dieses Ergebnis entspricht einem Stickstoff-Gehalt von 39,02\%.

Mit Salpetersäure eingedampft, gab der Rückstand nach dem Befeuchten mit Natronlauge eine deutliche Xantbin-Reaktion. Das mikroskopische Bild dieses Präparates war kein einheitliches und es lag offenbar ein Gemisch von verschiedenen Körpern vor. Es wurden daher $0,6 \mathrm{~g}$ dieser Substanz in $33 \mathrm{ccm}$ Wasser und $1 \mathrm{com}$ Natronlauge (14 $\mathrm{g}$ Natron und $86 \mathrm{~g}$ Wasser) gelöst und die Lösung mit $2 \mathrm{com}$ verdünnter Salzsäure (68 ccm konz. Salzsäure und $32 \mathrm{~cm}$ Wasser) versetzt, so daß die letztere im Überschusse vorhanden war. Es entstand ein Niederschlag, der sich selbst beim Erhitzen der Flüssigkeit bis zum Sieden nicht vollständig löste. Bei dem vorhandenen Überschusse von Salzsäure hätte sich die Substanz, falls sie aus Hypoxanthin bestanden hätte, leicht auflösen müssen. Nach mehrstündigem Stehen wurde der abfiltrierte und mit kaltem Wasser gewaschene Niederschlag nochmals derselben Operation unterworfen and schlieblich mit $30 \mathrm{ccm}$ Wasser und $0,5 \mathrm{ccm}$ verdünnter Salzsäure bis zum Sieden der Flüssigkeit erhitzt. Das Filtrat von der nach mehreren Stunden abfiltrierten, mit Wasser und Alkohol gewaschenen Substanz ergab nach dem Eindampfen derselben nur einen unbedeutenden Rückstand. Der auf die beschriebene Weise dargestellte, in verdünnter Salzsäure schwer lösliche Körper hinterließ nach dem Eindampfen mit konz. Salpetersäure einen gelben Fleck, der sich, mit Natronlauge befeuchtet, orangerot färbte. In ein Gemisch von Chlorkalk und Natronlauge eingetragen, bildete sich um die Körnchen ein grüner Hof. In Salpetersänre gelöst und mit Silbernitrat versetzt, entstand ein voluminöser Niederschlag, der sich beim Erwärmen in der überschüssigen Salpetersäure zum Unterschiede von dem Verbalten des Hypoxanthins leicht löste. Der Körper zeigte somit die Reaktionen des Xanthins, dessen Identitat durch das Ergebnis der folgenden Stickstoff-Bestimmung bestätigt erscheint.

$0,15 \mathrm{~g}$ Substanz verbrauchten nach $\mathrm{Kj}$ eldahl $7,93 \mathrm{~cm}$ 1/2 N.-Schwefelsäure; demnach in 100 Teilen:

$$
\begin{array}{ccc} 
& \text { Erbalten } & \text { Berechnet für } \mathrm{C}_{5} \mathrm{H}_{4} \mathrm{~N}_{4} \mathrm{O}_{2} \\
\text { Stickstoff } & 37,0 \% & 36,84 \%
\end{array}
$$

Die bei der Xanthin-Abscheidung sich ergebenden Filtrate und Waschwässer wurden vereinigt, mit Ammoniak im Überschub versetzt und bis auf ein kleines Vo- 
lumen eingedampft. Die abgeschiedene Substanz zeigte nur eine schwache XanthinReaktion, sonst aber das Verhalten von Hypoxanthin.

Der aus dem bleihaltigen, sehr schmutzig gefärbten Niederschlag $\mathbf{P} b_{2}$, durch Auflösen in siedend heifem Wasser unter Zusatz von Essigsäure und Eindampfen der mit Schwefelwasserstoff entbleiten Flüssigkeit sich ergebende Rückstand war stark schmutzig gefärbt und von einer schmierigen Beschaffenheit. Derselbe bedurfte einer weiteren Reinigung und ich habe deshalb die Xanthinkörper aus der Lösung dieses Rückstandes mit ammoniakalischer Silberlösung ausgefällt und aus dem erhaltenen Niederschlage durch Zersetzen desselben mit Schwefelwasserstoff in üblicher Weise die Xanthinbasen zurückgewonnen. Die gewonnene Substanz wog.0,4 g und wurde weiter durch Auflösen in verdünnter Natronlauge und Ausfällen aus dieser Lösung mit verdünnter Salzsäure gereinigt. Das erhaltene Präparat gab sämtliche Realitionen des Xanthins.

Die Fraktion A bestand somit vorwiegend aus Hypoxanthin und enthielt Xanthin nur in einer untergeordneten Menge. Das Xanthin war bei diesem Entfärbungsverfahren in den Bleiniederschlag übergegangen, während das Hypoxanthin größtenteils in Lösung blieb. Es ist jedoch notwendig, die Entfärbung mit Bleiessig unter Zusatz von Ammoniak bis zur Bildung eines Niederschlages stets in der Hitze vorzunehmen, da sonst größere Mengen von Hypoxanthin in den bleihaltigen Niederschlag übergehen. Ich habe mich dieses Verfahrens zur Reinigung der einzelnen Fraktionen mit Erfolg bedient. Aus den entbleiten Filtraten konnte ich die Xanthinstoffe unschwer in krystallisierter Form gewinnen. Die Anwesenheit der Essigsäure stört den Gang der Isolierung nicht, vielmehr scheint sie gewisse Stoffe, welche die sich ausscheidenden Xanthinbasen verunreinigen würden, in Lösung zu halten. Im übrigen ist dieselbe im Bedarfsfalle durch wiederholtes Eindampfen nicht schwer zu vertreiben. Dieses Entfärbungsverfahren, wenn auch umständlicher, ist der Entfärbung mit Tierkohle vorzuziehen, da man es viel leichter in der Hand hat, die Bleiniederschläge einer genaueren Prüfung zu unterziehen, als es bei der Tierkohle überhaupt möglich ist.

\section{Untersuchung der Fraktion B.}

Diese Fraktion verhielt sich gegen verdünntes Ammoniak, 3,3\%/0-ige Natronlauge und gegen Pikrinsäure wie die Fraktion A. Sie wurde deshalb nach demselben eben beschriebenen Bleiessigverfahren unter Zusatz von Ammoniak gereinigt, wie die Fraktion A. Die gewonnene Substanz entsprach in ihrem Verhalten gegen Reagentien vollkommen dem Hypoxanthin. Auch das Ergebnis der folgenden Analyse beweist die Identität des Präparates mit Hypoxanthin:

0,2000 g Substanz verbrauchten $11,77 \mathrm{ccm}$ 1/2 N.-Schwefelsäure, $0,2772 \mathrm{~g}$ " gaben $0,0714 \mathrm{~g}$ Wasser und $0,4520 \mathrm{~g}$ Kohlensäure; demnach:

$\begin{array}{lccc} & \text { Stickstoff } & \text { Kohlenstoff } & \text { Wasserstoff } \\ \text { Erhalten . } . * . & 41,19 \% & 44,47 \% & 2,86 \% \\ \text { Berechnet für } \mathrm{O}_{5} \mathrm{H}_{4} \mathrm{~N}_{4} \mathrm{O} . & 41,18 \% & 44,12 \% & 2,94 \%\end{array}$

Der bei der Reindarstellung dieses Präparates erhaltene Bleiniederschlag wurde in derselben Weise wie der Niederschlag $\mathrm{Pb}_{2}$ der Fraktion $\mathrm{A}$ behandelt. Die aus dem silberhaltigen Niederschlage gewonnene Substanz wog $1,0 \mathrm{~g}$. Dieselbe wurde in das salpetersaure Salz übergeführt, welches, mikroskopisch untersucht, die Formen des 
salpetersauren Hypoxanthins zeigte. Aus der mit Ammoniak versetzten Lösung dieses Salzes wurde die freie Base durch Eindampfen auf ein kleines Volumen gewonnen und bei $130^{\circ} \mathrm{C}$. getrocknet.

$0,2 \mathrm{~g}$ dieser Substanz verbrauchten nach $\mathrm{Kjeldahl} 11,6 \mathrm{ccm}{ }^{1 / 2} \mathrm{~N}$.-Schwefelsäure. Aus diesem Ergebnis berechnet sich ein Stickstoffgebalt von $40,6 \%$ gegenübex $41,18 \%$ Stickstoff in Hypoxanthin.

Die fragliche Substanz bestand offenbar hauptsächlich aus Hypoxanthin, war jedoch nicht vollständig rein, da sie einen Stich ins Schmutzig-gelbliche hatte. Daraus erklärt sich der etwas niedrige Gehalt des Präparates an Stickstoff.

Die Waschwässer von den Hypoxanthin-Krystallisationen, welche die Hauptmenge der Fraktionen A und B ausmachte, wurden vereinigt und aus denselben die Xanthinkörper mit ammoniakalischer Silberlösung ausgefällt. Die aus diesem Silberniederschlage in üblicher Weise gewonnene Substanz wog 1 g. Dieselbe wurde in das salpetersaure Salz übergefübri und aus diesem die freie Base in der sehon wiederholt angegebenen Weise dargestellt.

$0,2 \mathrm{~g}$ des bei $130^{\circ} \mathrm{C}$. getrockneten Präparates verbrauchten nach Kjeldah」11,7 com

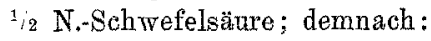

Stickstoff $\begin{gathered}\text { Erhalten } \\ 40,95 \%\end{gathered} \quad$ Berechnet für Hypoxanthin $\mathrm{C}_{5} \mathrm{H}_{ \pm} \mathrm{N}_{4} \mathrm{O}$

Die Eigenschaften dieses Präparates und die Krystallformen seines salpetersauren Salzes entsprachen denen des Hypoxanthins.

\section{Untersuchung der Fraktion C.}

Die bis zum Sieden erhitzte Lösung von je $10 \mathrm{~g}$ Substanz in $200 \mathrm{ccm}$ Wasser wurde mit 1,10;0-iger Pikxinsäurelösung in mäBigem Uberschuß verset $z$ t und nach dem langsamen Abkühlen auf Zimmertempexatur der entstandene Niederschlag (Fraktion D) abfiltriert und derselbe mit möglichst wenig Wasser gewaschen, in heißer verdünnter Salzsäure gelöst und aus der noch warmen Lösung die Pikrinsäure mil Toluol ansgeschüttelt. Ammoniak in mäBigem Uberschusse der salzsauren Flüssigkeit zugesetzt, erzeugte selbst nach 6-stündigem Stehen keinen Niederschlag. Aus dieser Lösung wurden darauf die Xanthinstoffe mit ammoniakalischer Silberlösung gefällt und aus dem entstandenen Niederschlage als freie Basen dargestellt. Das bei dieser Behandlung sich ergebende Schwefelsilber gab an heibe verdünnte Salzsäure keine Xanthinstoffe ab.

Da die gewonnene Substanz, welche $3,3 \mathrm{~g}$ wog, nicht genügend rein war und sich durch Krystallisation nicht gut reinigen ließ, habe ich dieselbe behufs weiterer Reindarstellung dem schon öfters erwähnten Bleiessigverfahren unterworfen. Der erzeugte Bleiniederschlag enthielt nur eine geringfügige Meage aus ammoniakalischer Silberlösung fällbarer Substanzen. Das Filtrat rom Schwefelblei enthielt freie Essigsäure, welche aus dem überschüssig zugesetzten Bleiessig stammte und insofern auf die Krystallisation der Substanz, welche offenbar aus A denin bestand, störend wirkte, als sie dieselbe in Lösung hielt. Es wurde daher die Flüssigkeit beim Eindampfen mit Ammoniak in mäßigem .Überschuß versetzt. Aus der eingeengten, fast neutralen Lösung konnte das Adenin leicht in langen Nadeln, bezw. Prismen krystallisiert erhalten werden. Dasselbe wurde zunächst in siedendem Alkohol gelöst, wobei nur ein geringfügiger unlöglicher Rückstand zurückblieb. 
Nach dem Abdestillieren des Alkohols wurde die Substanz aus Wasser umkrystallisiert. Das erhaltene, schön- krystallisierte Präparat zeigte alle Eigenschaften des Adenins und auch das Ergebnis der folgenden Analyse beweist die Identität dieser Substanz mit Adenin.

$0,2000 \mathrm{~g}$ Substanz verbrauchten nach Kjeldahl 14,85 $\mathrm{ccm}^{1 / 2}$ N.-Schwefelsäure, $0,2758 \mathrm{~g} \quad, \quad$ gaben $0,091 \mathrm{~g}$ Wasser und $0,4514 \mathrm{~g}$ Kohlensäure; demnach:

\begin{tabular}{|c|c|c|c|}
\hline & Stickstoff & Kohlenstoff & Wasserstoff \\
\hline & $51,97 \%$ & $44,64 \%$ & $3,67 \%$ \\
\hline Berechnet für Adenin $\left(\mathrm{C}_{5} \mathrm{H}_{5} \mathrm{~N}_{\overline{3}}\right)$ & 51,85 & 44,45 , & 3,70 \\
\hline
\end{tabular}

Aus dem auf ein entsprechendes Volumen eingedampften und mit Salzsäure versetzten Filtrate vou der Fraktion D wurde die Pikrinsäure mit Toluol ausgeschüttelt. Aus der salzsauren Lösung wurden die Xanthinkörper mit Hilfe ihrer Silberverbindungen durch Zersetzen der letzteren mit Schwefelwasserstoff als freie Basen abgeschieden. Beim Einengen des Filtrates auf ein kleines Volumen schied sich eine Substanz (Fraktion E) ab, welche nach dem Abfiltrieren, Waschen mit Wasser und Alkohol und Trooknen bei $100^{\circ}$ C. $14 \mathrm{~g}$ betrug. Dieselbe wurde nach dem bereits bekannten Bleiessigverfahren einer weiteren Reinigung unterzogen. Das Präparat zeigte die Eigenschaften des Hypoxanthins, dessen Identität durch die folgenden StickstoffBestimmungen bestätigt erscheint.

Je $0,2 \mathrm{~g}$ Substanz verbrauchten nach Kjeldahl 11,80 bezw. $11,85 \mathrm{ccm}^{1 / 2}$ N.-Schwefelsäure; demnach :

\section{Erbalten}

Stickstoff

\section{1,47 und $41,30 \%$}

\section{Berechnet fïr Hypoxanthin $\left(\mathrm{O}_{\overrightarrow{5}} \mathrm{H}_{4} \mathrm{~N}_{4} \mathrm{O}\right)$} $41,18 \%$

Aus dem bei der Reindarstellung dieses Präparates sich ergebenden Bleiniederschlage wurden $0,55 \mathrm{~g}$ und aus den Waschwässern von der Krystallisation des eben analysierten Präparates $0,65 \mathrm{~g}$ einer Substanz gewonnen. Beide Substanzen dürften ihrem Verhalten nach gegen Reagentien vorwiegend aus Hypoxanthin bestehen.

Durch abermaliges Einengen des Filtrates von der Fraktion $\mathbf{E}$ erhielt ich noch $0,1 \mathrm{~g}$ einer unlöslichen Substanz, die ich wegen der geringen Menge einer weiteren Untersuchung nicht mehr unterzog. Das Filtrat wurde nun zur Trockene gebracht und mit siedendem Alkohol unter Zusatz einiger Tropfen Essigsüure extrahiert. Der in Alkohol unlösliche Rückstand war stark aschenhaltig, erwies sich dagegen als frei von Xanthinkörpern, welche in die alkoholische Lösung übergegangen sind. Nach dem Abdestillieren des Alkobols wurde der Rückstand in heißem Wasser aufgenommen und die Lösung mit Pikrinsäure in mäßigem Überschuß versetzt. Der nach langsamen Abkühlen auf Zimmertemperatur entstandene Niederschlag (Fraktion F) wurde in derselben Weise wie die Fraktion $D$ verarbeitet und exgab $0,3 \mathrm{~g}$ in Nadeln krystallisiertes Adenin. Andere Xanthinstoffe als Adenin fanden sich in dem PikrinsäureNiederschlage nicht vor.

Das Filtrat von der Fraktion $F$ behandelte ich in derselben Art wie das von der Fraktion D. Nach dem Eindampfen des Filtrates von Schwefelsilber schied sich $0,64 \mathrm{~g}$ Substanz aus, die, nach den Bleiessigverfabren gereinigt, 0,52 $\mathrm{g}$ wog. Dieses Präparat zeigte alle Eigenschaften, die dem Hypoxanthin eigen sind. Der Gehalt des Präparates an Stickstoff entspricht, wie aus dem Ergebnisse der folgenden Analyse hervorgeht, dem des Hypoxanthins. 
$0,2 \mathrm{~g}$ der bei $130^{\circ} \mathrm{O}$. getrockneten Substanz verbrauchten nach Kjeldahl $11,8 \mathrm{ccm}$ 1/2 N.-Schwefelsäure; demnach:

$$
\text { Stickstoff } \begin{gathered}
\text { Erhalten } \\
41,30 \%
\end{gathered} \quad \text { Berechnet für Hypoxanthin }\left(\mathrm{C}_{5} \mathrm{H}_{4} \mathrm{~N}_{4} \mathrm{O}\right)
$$

Das Filtrat von der letzten Fraktion ergab nach dem Verdunsten ungefähr $1 \mathrm{~g}$ eines unreinen Rückstandes, in dessen wässeriger Lösung Pikrinsäure einen hellgelben Niederschlag, der aus pikrinsaurem Adenin bestehen dürfte, erzeugte. Ich habe diesen letzten Rückstand, als nicht mehr der Mühe lohnend, nicht näher untersucht.

Aus dieser Untersuchung erhellt, da $B$ das Hypoxanthin den vorwiegenden Teil der aus dem Fleischextrakte nach dem angeführten Verfahren abgeschiedenen Xanthinkörperbildet. Das Xanthin ist gegenüber dem Hypoxanthin im Fleischextrakte in einer nur untergeordneten Menge enthalten. Die angeführte Menge Xanthin dürfte etwas zu niedrig sein. Denn durch nachträgliche Untersuchung der eingangs erwähnten aus der Tierkohle, welche zur Entfärbung der Lösungen dex salzsauren Xanthinkörper diente, durch Extraktion mit heißer verdünnter Salzsäure erhaltenen Xanthinbasen, konnte ich unter diesen das Xanthin in bestimmbaren Mengen nachweisen. Deshalb wird an dem Gesamt-Ergebnisse dieser Untersuchung nichts oder nur wenig geändert. Im übrigen habe ich zur besseren quantitativen Bestimmung des Xanthins und auch der anderen vorgefundenen Xanthinkörper eine neue Menge von Fleischextrakt in Arbeit genommen, über welche Untersuchungen ich später zu berichten gedenke.

AuBer Hypoxanthin und Xanthin wurde noch das Adenin nachgewiesen, welches bis jetzt im Fleischextrakte noch nicht rorgefunden worden zu sein scheint. Aus diesem Befunde geht auch hervor, daß bei der Bereitung des Fleischextraktes nicht sämtliche Xanthinbasen des Muskels in den Extrakt übergehen. Ein Beispiel hierfür bildet das $\mathrm{G}$ u a $\mathrm{n}$ in, welches von $\mathrm{Kosse}^{1}$ ) im Rindsmuskel zu $0,09 \%$ bestimmt wurde. Unter den Xanthinbasen des Fleischextraktes konnte ich Guanin nicht nachweisen.

Auffälig muB es jedoch erscheinen, daB das Karnin bei diesen Untersuchungen des Fleischextraktes auf Xanthinkörper nicht vorgefunden wurde. Das Karnin bildet nach der Beschreibung seiner Eigenschaften in der Literatur mit ammoniakalischer Silberlösung eine in Ammoniak unlösliche Verbindung. Auf Grund dieser und anderer bekannten Eigenschaften des Karnins wäre es schwer möglich, daß sich das Karnin bei der vorgenommenen Untersuchung dem Nachmeise hätte entziehen konnen, um so weniger, als nach $W$ eidel das Karnin im Fleischextrakt zu $1 \%$ enthalten sein soll. Da ich $3 \mathrm{~kg}$ Extrakt verarbeitete, hätten aus dieser Menge $30 \mathrm{~g}$ Karnin sich ergeben müssen. Eine solche Menge einer Substanz ließe sich bei der beschriebenen Untersuchung nicht übersehen. Bei der nicht vollständigen Kenntnis des Karnins könnte vielleicht der Einwand erhoben werden, daß das Karnin mit Kupferbisulfit nicht gefällt wurde. Mit den vorstehenden Untersuchungen beschäftigt, habe ich von den Filtraten des Kupferbisulfitniederschlages und auch von den Filtraten des Silberniederschlages Proben entnommen und dieselben auf Xanthinbasen geprüft. Allein weder in dem einen noch in dem anderen konnten Xanthinstoffe nachgewiesen werden. Zum Überflusse habe ich schließlich das gesamte Filtrat samt den Waschwässern von aus $300 \mathrm{~g}$ verarbeitetem Extrakt erhaltenem Kupferbisul-

1) Zeitschr. physiol. Chem. 1884, 8, 404. 
fit-Niederschlag in folgender Weise auf Xanthinstoffe geprüft. Die Flüssigkeit wurde mit verdünnter Schwefelsäure behufs Zersetzung der überschüssigen Bisulfitsalze versetzt, auf ein entsprechendes Volumen eingedampft und mit Schwefelwasserstoff entkupfert. Das Filtrat vom Schwefelkupfer wurde noch weiter eingeengt und mit Ammoniak in mäBigem ÜberschuB vermischt. Es bildete sich ein weiBer Niederschlag, der wie die nähere Untersuchung ergab, aus phosphorsaurem Kalk bestand. Auf Zusatz von ammoniakalischer Silberlösung zu dem Filtrate von phosphorsaurem Kalk entstand nach 24-stündigem Stehen ein verhältnismäßig geringer, feiner und dunkel gefärbter Niederschlag, der also nicht die Beschaffenheit hatte, wie dies bei Anwesenheit von Xanthinstoffen der Fall ist. Da dieser Silberniederschlag, der teils aus Schwefelsilber, teils aus reduziertem Silber bestehen dürfte, sich nicht gut abfiltrieren lief, inden das Filtrat stets trüb erschien, wurde derselbe aus der Flüssigkeit auszentrifugiert und mit stark verdünntem Ammoniak, dann mit reinem Wasser unter Anwendung der Centrifuge gewaschen. Dieser Niederschlag in der üblichen Weise geprüft, enthielt keine Xanthinbasen. Das Kupferbisulfit hat also die mit ammoniakalischer Silberlösung fällbaren Xanthinstoffe vollständig ausgefällt.

Das bei der Verarbeitung des Kupferbisulfit-Niederschlages abfallende Schwefelkupfer wurde mit siedend heißer, verdünnter Salzzäure ausgezogen und das teilweise in Lösung gegangene Kupfer mit Schwefelwasserstoff wieder ausgefällt. In dem eingeengten und mit Ammoniak im Überschul versetzten Filtrate erzeugte eine ammoniakalische Silberlösung nur einen unbedeutenden Niederschlag. Es waren somit in dem Schwefelkupfer-Niederschlage keine nennenswerten Mengen Xanthinkörper vorhanden.

Der aus 300 g Extrakt gewonnene Xanthinbasen-Silber-Niederschlag war auf 2 Filtern verteilt. Die vereinigten Filtrate und die Waschwässer von denselben betrugen ungefähr $8 \mathrm{l}$. Aus dieser gesamten Flüssigkeit fällte ich das Silber durch Einleiten von Schwefelwasserstoff. Das Filtrat von Schwefelsilber wurde bis zur Salzbildung eingedampft und der Abdampfrückstand erst nach 24-stündigem Stehen in kaltem Wasser aufgenommen. Es blieb nur eine geringe Menge einer unlöslichen Substanz zurück, die sich bei näherer Untersuchung als Schwefel erwies. Die Lösung des Abdampfrückstandes gab auf Zusatz von ammoniakalischer Silberlösung und nach einer Stunde, eine Trübung, die sich nach weiterem Stehen langsam zu einem ganz unbedeutenden Niederschlag absetzte. Es waren demnach in der beträchtlichen Menge der Filtrate bezw. der Waschwässer nur unwesentliche Mengen Xanthinkörper nachweisbar.

Das bei der Zersetzung des obigen Silberniederschlages der Xanthinbasen mit Schwefelwasserstoff sich ergebende Schwefelsilber wurde mit heißer verdünnter Salzsäure behandelt. In diesem salzsauren Auszuge waren keine Xanthinkörper enthalten.

Fragt man sich nach dem Verbleiben von Karnin, dann gibt es zwei Möglichkeiten. Entweder ist das Karnin eine derart zersetzliche Verbindung, daß sie der Einwirkung der kochenden verdünnten Schwefelsäure nicht Stand hält, und in diesem Falle sehr wahrscheinlich Hypoxanthin, welches nach Weidel durch Oxydation des Karnins mit Brom oder Salpetersäure entsteht, als Spaltungsprodukt liefert, oder es war in dem vorliegenden Extrakte kein Karnin enthalten. Die erstere Möglich-

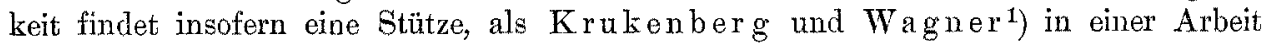

1) Sitzungsberichte der Würzburger med.phys. Gesellsch. 1883; Ref. in Ber. Dentsch. Chem. Gesellsch. 1885, 18, Ref. 641. 
"Über Besonderheiten des chemischen Baues kontraktiler Gewebe" in einzelnen Portionen Fleischextrakt statt Karnin Hypoxanthin fanden und diesen Befund einer Spaltung des vorhandenen Karnins zuschrieben.

Um die diesbezügliche Frage näher zu beantworten, habe ich eine weitere Menge des Fleischextraktes in Arbeit genommen, um aus demselben das Karnin genau nach der Vorschrift von Weidel ${ }^{1}$ ) darzustellen.

1) Annal. Chem. u. Pharm. 168, 353.

\section{Referate.}

\section{Ernährungslehre.}

William J. Gies: Eine verbesserte. Methode der Präparation und Konservierung von Fleisch, das zu. Stoffwechselversuchen dienen soll. (Americ. Journ. Physiol. 1901, 5, 235-239.) - Zur Herstellung von größeren Mengen Fleisch, das zu Stoffwechselversuchen an Tieren Verwendung finden soll, bedient sich Verf. des folgenden Verfahrens: Nachdem das magere Fleisch von dem anhaftenden Fett, Sehnen u. s. w. befreit worden ist, wird es fein gehackt und in Mengen von $6-10 \mathrm{~kg}$ unter einer starken Fleischpresse so lange gepreßt, bis Blut enthaltende Flüssigkeit aus dem Fleischbrei nicht mehr abläuft. Nachdem darauf Proben zur Analyse der Fleischmasse entnommen sind, wird dieselbe mit den Händen $\mathrm{zu}$ Ballen von je 50-100 g Gewicht gerollt, je 5-6 dieser Klößchen in weithalsige Kölbchen gebracht und diese in einen Kälteraum gestellt, in dem eine Temperatur von $0^{0}$ oder darunter herrscht. Nach kurzer Zeit sind die Fleischklöße gefroren, infolge dessen kann Flüssigkeit aus der Masse fernerhin nicht mehr austreten. Das nach diesem Verfahren behandelte Fleisch bält sich lange frisch und besitzt, wie Verf. durch Versuche nachgewiesen hat, noch nach 30 Tagen dieselbe Zusammensetzung wie am Tage seiner Herstellung, ein Unstand, der bei länger währenden Stoffwechselversuchen von groBer Bedeutung ist.

Max Miller.

K. B. Lehmann: Über die Bedeutung der Zerkleinerung und des Kochens der Speisen für die Verdaung. (Arch. Hyg. 1902, 43, 123-133.) - Verf. versuchte festzustellen, welchen Einfluß der Grad der Zerkleinerung auf die Verdauung besitze. $\mathrm{Zu}$ diesem Zweck wurde eine Reihe von Versuchen mit Hühnereiweib, Fleisch, Käse und Kohlenhydraten angestellt. Bei letzteren wurden die Versuche nach 2 Richtungen hin unternommen, indem erstens die Auslaugung wasserlöslicher Zuckerarten aus rohen und gekochten pflanzlichen Nahrungsmitteln (Äpfeln und gelben Rüben) bestimmt und zweitens die Bildung von Zucker aus rohen und gekochten stärkereichen pflanzlichen Nabrungsmitteln (jungen italienischen Kartoffeln und Maccaroni) bei verschiedener Zerkleinerung untersucht wurde. Die Ergebnisse der Arbeit zeigen nun aufs klarste die Bedeutung der Zerkleinerung für die Lösung und Verdauung von Eiweißkörpern und Kohlenhydraten unserer Nahrung. Besonders wichtig erscheint, daß meist zwischen der mittelfeinen (etwa $1 \mathrm{~mm}$ ) Zerkleinerung and der feinsten Zerreibung noch ein erheblicher Unterschied besteht. - In der Mehrzahl der Versuche stellten die Werte der Lösungszahlen von Eiweißkörpern oder Kohlehydraten bei der Zerkleinerung zu etwa $1 \mathrm{~mm}$ großen Stückchen ungefähr das Mittel dar zwischen denen, die bei grober Zerkleinerung und feinster Zerreibung erhalten wurden. 